\title{
Maternal stress level when a baby is admitted to the neonatal intensive care unit at Teaching Hospital Jaffna and the influence of maternal and infant characteristics on this level
}

\author{
*Nirubaa Umasankar ${ }^{1}$, Manoji Gitanjali Sathiadas ${ }^{2}$ \\ Sri Lanka Journal of Child Health, 2016; 45(2): 90-94
}

\begin{abstract}
Background: Currently, little is known about the maternal stress when a neonate is admitted to a Neonatal Intensive Care Unit (NICU) in Sri Lanka.

Objective: To assess the maternal stress level when a baby is admitted to the NICU at Teaching Hospital Jaffna (THJ) and the influence of maternal and infant characteristics on this level.

Method: A prospective cross sectional descriptive study was conducted in NICU at THJ on 75 motherbaby pairs using the Parental Stressor Scale: Neonatal Intensive Care Unit to measure the maternal stress level. A parent/infant demographic sheet provided information for determining which characteristic resulted in a different response.
\end{abstract}

Results: Seventy six percent of mothers experienced severe and extreme stress level for overall stress. The mean for overall stress experienced by the mothers was $4.01 \pm 0.87$. The highest levels of stress experienced were in the sight and sound subscale with a mean of $3.04 \pm 1.04$ and the relationship with the baby-parental role (mean $2.93 \pm 1.28$ ). The infant characteristic of gestational age resulted in a significant different score concerning the appearance and behaviour of the baby, even though there were no significant differences in the score experienced in the overall stress level. There was no significant difference in the overall stress score with maternal characteristics such as age, parity, maternal level of

${ }^{1}$ Lecturer in Paediatrics, Honorary Consultant Paediatrician, ${ }^{2}$ Head and Senior Lecturer in Paediatrics, Honorary Consultant Paediatrician, Faculty of Medicine, University of Jaffna

*Correspondence: unirubaa@yahoo.com

(Received on 12 August 2015: Accepted after revision on 18 September 2015)

The authors declare that there are no conflicts of interest

Personal funding was used for this project.

Open Access Article published under the Creative Commons Attribution CC-BY (cc) (P) education and income, but a previous baby being admitted to the NICU had a significant correlation with the overall stress level $(p=0.04)$.

Conclusions: Mothers suffer significantly high stress levels when their babies need NICU admission. NICU physical environment and alteration in parental role are the main sources of stress. Infant characteristics like gestational age, birth asphyxia and sepsis are related to high maternal stress levels. Previous baby needing NICU admission and previous neonatal deaths have strong associations with high maternal stress levels.

DOI: http://dx.doi.org/10.4038/sljch.v45i2.8003

(Key words: maternal stress, NICU, maternal characteristics, infant characteristics, parental stress scale)

\section{Introduction}

Advances in medical knowledge and technology have made babies on the edge of viability to be cared for and kept alive. However, for parents, birth of an infant requiring admission to the Neonatal Intensive Care Unit (NICU) can represent a considerable source of stress. Appearance of the baby and uncertainty about outcome are potential sources of stress. The NICU environment has the potential to exacerbate this stress. NICU stressors, individually or in combination, may interfere with the mother-baby relationship and thus affect infant development subsequently. Currently, little is known about the maternal stress when neonates are admitted to NICUs in Sri Lanka.

\section{Objective}

To assess the maternal stress level when a baby is admitted to the NICU at Teaching Hospital Jaffna and the influence of maternal and infant characteristics on this level.

\section{Method}

This was a prospective cross sectional descriptive study conducted in NICU at Teaching Hospital Jaffna. Sample size was calculated by using studies done in other parts of the world, and the study was 
conducted from $1^{\text {st }}$ June 2014 till calculated sample size of 75 mother-baby pairs was obtained. Data was collected using the questionnaire from each study unit. Self-administered questionnaire was translated into Tamil and validated before starting the study. Parent/infant pair demographic details were collected by interviewer administered questionnaire and details from the bed head tickets. All the data were entered and analysed using the software SPSS version 16.

The Parental Stressor Scale: Neonatal Intensive Care Unit (PSS: NICU) by Miles ${ }^{1}$ was used to measure the maternal stress level after getting permission from the author. The PSS: NICU contains 46 items, corresponding to four subscales viz. NICU environment, infant appearance and behaviour, parent infant relationship, staff behaviour and a general stress item. Participants were asked to rate each item, according to how stressful the situation described in each item was for them: $1=$ not at all stressful, $2=\mathrm{a}$ little stressful, $3=$ moderately stressful, $4=$ very stressful, and $5=$ extremely stressful. Stress was defined as feeling anxious, upset or tense. If participants had not experienced a particular situation, they were asked to indicate this by answering "not applicable".

Ethical clearance was obtained from the Ethical Review Committee of the Sri Lanka College of Paediatricians.

\section{Results}

Seventy five mother-baby pairs were studied. Table 1 demonstrates the maternal characteristics of the study population.

Table 1: Maternal characteristics of study population

\begin{tabular}{|l|c|}
\hline \multicolumn{1}{|c|}{ Maternal characteristic } & No. (\%) \\
\hline Parity & \\
Primipara & $38(51)$ \\
Multipara & $37(49)$ \\
\hline Maternal age & \\
$<20$ years & $03(04)$ \\
20-29 years & $33(44)$ \\
30-39 years & $39(52)$ \\
\hline Mode of delivery & \\
Normal vaginal & $38(51)$ \\
Elective caesarean section & $23(31)$ \\
Emergency caesarean section & $10(13)$ \\
Instrumental & $04(05)$ \\
\hline Previous baby needing NICU admission & $09(12)$ \\
\hline Previous neonatal death & $07(09)$ \\
\hline
\end{tabular}

Table 2 demonstrates the infant characteristics of the study population.
Table 2: Infant characteristics of study population

\begin{tabular}{|l|c|}
\hline \multicolumn{1}{|c|}{ Infant characteristic } & No. (\%) \\
\hline Gestational age & \\
$<30$ completed weeks & $03(04)$ \\
31 completed weeks & $04(05)$ \\
32 completed weeks & $11(15)$ \\
$33-36$ completed weeks & $10(13)$ \\
$>36$ completed weeks & $47(63)$ \\
\hline Birth weight & \\
$<1000 \mathrm{~g}$ & $01(01)$ \\
$1000-1500 \mathrm{~g}$ & $13(17)$ \\
$1501-2500 \mathrm{~g}$ & $27(36)$ \\
$>2500 \mathrm{~g}$ & $34(46)$ \\
\hline Age at admission to NICU & \\
At birth & $46(62)$ \\
Within $1^{\text {st }}$ week of life & $22(29)$ \\
During $2^{\text {nd }}$ week of life & $03(04)$ \\
During $3^{\text {rd }}$ week of life & $01(01)$ \\
In $4^{\text {th }}$ week of life & $03(04)$ \\
\hline
\end{tabular}

Mean birth weight of the babies was 2326.7 $\pm 781.6 \mathrm{~g}$. Mean age of admission to NICU was $3.21 \pm 5.6$ days.

Table 3 demonstrates the reasons for admission to NICU

Table 3: Reasons for admission to NICU

\begin{tabular}{|l|c|}
\hline \multicolumn{1}{|c|}{ Reason } & No. of babies (\%) \\
\hline Low birth weight & $39(52.0)$ \\
\hline Prematurity & $29(38.7)$ \\
\hline Respiratory problems & $23(30.7)$ \\
\hline Sepsis & $22(29.3)$ \\
\hline Birth asphyxia & $07(09.3)$ \\
\hline Jaundice & $08(10.7)$ \\
\hline Convulsions & $05(06.5)$ \\
\hline Weight loss & $06(07.8)$ \\
\hline Fever & $12(15.6)$ \\
\hline
\end{tabular}

Multiple responses were included

Figure 1 demonstrates the duration of NICU stay.

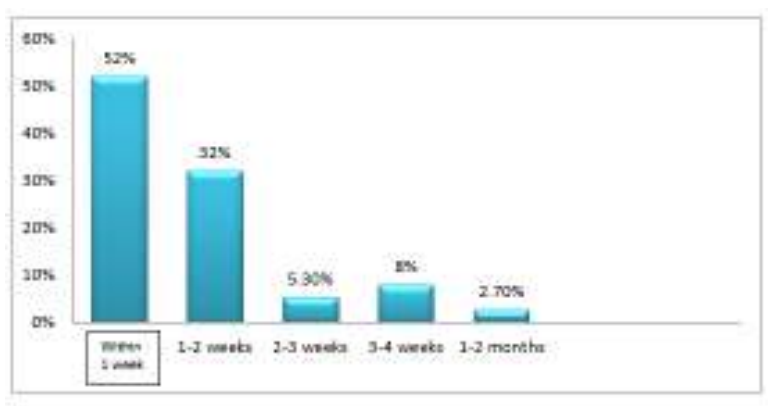

Figure 1: Duration of NICC stay

The mean duration of stay at NICU was $10 \pm 9$ days. 
Figure 2 indicates the outcome of the baby.

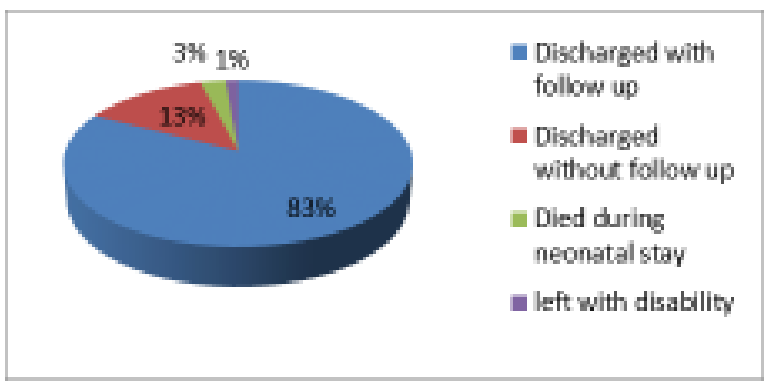

Figure 2: Outcome of the baby

Figure 3 demonstrates the overall stress level of the mothers.

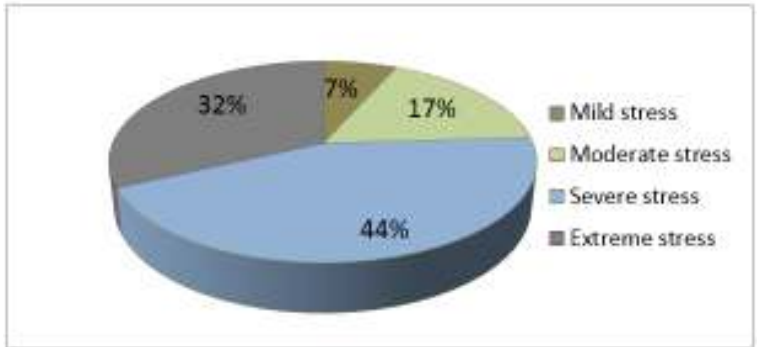

Figure 3: Distribution of overall stress level of mothers

The mean stress score for overall stress was $4.01 \pm$ 0.87 which falls in the category of severe stress.

Table 4 demonstrates the stress caused by the subscales.

Table 4: The stress caused by the different subscales of PSS: NICU

\begin{tabular}{|l|c|c|c|c|c|c|}
\hline \multicolumn{1}{|c|}{ Subscale of stress } & $\begin{array}{c}\text { Not } \\
\text { applicable }\end{array}$ & $\begin{array}{c}\text { Not } \\
\text { stressful }\end{array}$ & $\begin{array}{c}\text { Mildly } \\
\text { stressful }\end{array}$ & $\begin{array}{c}\text { Moderately } \\
\text { stressful }\end{array}$ & $\begin{array}{c}\text { Severely } \\
\text { stressful }\end{array}$ & $\begin{array}{c}\text { Extremely } \\
\text { stressful }\end{array}$ \\
\cline { 2 - 6 } & \multicolumn{7}{|c|}{$(\mathrm{N}=75)$} \\
\hline Sight and sound & 0 & $23 \%$ & $21 \%$ & $32 \%$ & $21 \%$ & $3 \%$ \\
\hline Behaviour of the baby & $17 \%$ & $21 \%$ & $31 \%$ & $16 \%$ & $12 \%$ & $3 \%$ \\
\hline Alteration in parental role & $8 \%$ & $13 \%$ & $25 \%$ & $31 \%$ & $21 \%$ & $2 \%$ \\
\hline Staff behaviour & $47 \%$ & $21 \%$ & $12 \%$ & $12 \%$ & $7 \%$ & $1 \%$ \\
\hline
\end{tabular}

Comparison of stress caused by different subscales with studies done in other parts of the world is shown in Figure 4.

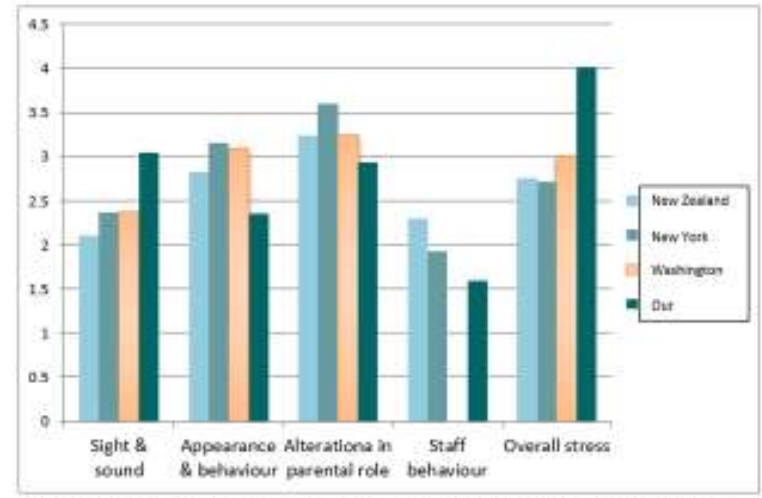

Figure 4: Comparison of stress caused by different subscales with studies done in other parts of the world

The above diagram shows the overall stress level and the stressors caused by different subclasses in studies done in New Zealand, New York, Washington and comparison with our study ${ }^{2-4}$. The overall stress level is very high in our study when compared to other studies. When identifying the main source of stress, the highest stress caused in all other studies was due to alteration in the parental role, but in our study it is due to sight and sound of the NICU. The second commonest cause of stress in our study is alteration in the parental role and the stress levels are comparable to other studies. The stress caused by appearance and behaviour of the baby is less in our study when compared to other studies because most of the mothers in our study had not experienced that stressful event.

Correlation of the maternal stress level with different infant and maternal characteristics

When analysing the relationship of maternal stress level with different infant characteristics, the stress caused by appearance and behaviour of the baby is significantly high when the gestational age is low $(p=0.012)$ and when the baby is admitted with sepsis $(p=0.036)$. The stress caused by alteration in the parental role is significantly high when the baby is in the incubator $(\mathrm{p}=0.017)$ and when the baby is asphyxiated. The levels are significantly low when the baby is in the mother-baby side $(\mathrm{p}=0.001)$ and when the baby is needing admission to NICU beyond 24 hours after birth $(p=0.001)$. When the duration of stay is high the stress levels are significantly high in the sight and sound, behaviour of the baby and staff behaviour ( $p=0.001,0.001$ and 0.025 ). The stress levels are high when there is a history of previous baby needing NICU admission and there is a significant correlation $(p=0.039)$. Stress levels are significantly high in all aspects if there is a previous neonatal death. The $\mathrm{p}$ values for sight and sound, baby's appearance and behaviour, alteration in the 
parental role and staff behaviour are $0.012,0.012$, 0.025 , and 0.019 respectively.

\section{Discussion}

Parental stress resulting from experience with infants hospitalised in NICU is well documented ${ }^{2-6}$. This study shows, on an overall basis, that mothers feel severe stress when their baby is admitted to the NICU in contrast to other studies, where the overall stress level falls into the mild to moderate range ${ }^{2-4}$. Especially with regard to the different areas of stress measured by the PSS: NICU, results of this study are different from other studies $^{2-4}$ where the most stressful aspects of having an infant in the NICU are altered parent role and relationship with their baby. However, in this study the most stressful area was the NICU physical environment. The stress caused by the environmental factor (sight and sound component in the PSS: NICU) was unexpectedly very high in our study. In other studies this component contributes only to mild stress but in our study it contributed to a moderate stress level. This finding is very important for our intervention, because the higher overall stress level may be due to high environmental stress in our study. In most parts of the world the mothers are usually introduced to the hospital and the ward setup. Both parents are encouraged to visit the NICU and an explanation is given by the NICU staff about the technology and the equipment which is connected to the baby. This makes the mothers more comfortable and may reduce stress caused by the intensity of the environment and also may be effective in reducing the overall stress of the mothers. Altered parental role is the second common cause of stress in our study. Inability to breast feed their baby, inability to carry the baby whenever they like, do the daily needs of their baby on their own and not being able to share the baby with the relatives are the main aspects in the altered parental role. Stress due to altered parental role is significantly high when the baby is in the incubator $(p=0.017)$ and significantly low when the baby is admitted with the mother in the mother-baby side $(\mathrm{p}=0.001)$.

Each of these findings is very important for us to give support to the mothers to facilitate a positive motherbaby relationship. Recognising their infant's cues and method of handling their babies should be emphasized to mothers during visit to the NICU. We have to provide support for care giving procedures that are appropriate for the mother and the infant's level of tolerance. Encouraging kangaroo mother care when the baby is stable in incubator and educating the mothers on cup feeding and getting them to cup feed their babies will be helpful. These interventions may reduce the stress due to altered parental role and facilitate mother baby bonding.

The least stressful area for parents in this study was staff behaviour and communication which is similar to other studies ${ }^{2-4}$. The stress caused by staff behaviour is high when there is a longer duration of stay at NICU. This is similar to other studies too. This indicates that the health care providers have to provide regular communication with mothers throughout the length of their NICU stay in order to reduce this. This study also indicates that some infant and maternal characteristics are more correlated to specific subscales of the stress rather than overall stress level. For instance, mothers of premature babies are more stressed due to altered appearance and behaviour of the baby whilst mothers of asphyxiated babies are worried due to altered parental role. The stress levels are significantly high when there is a previous baby needing NICU stay or previous neonatal death. Thus we have to communicate with mothers explaining the relevant aspects to reduce their stress levels. Finally, to apply these findings into practice, each of these findings can vary depending on environment of the NICU. Thus we have to analyze stress factors in each NICU and provide the support needed to facilitate a positive mother infant relationship.

Future research using a larger sample of parents in different NICUs, a more detailed and continuing communication with mothers regarding their baby's condition and involvement of the mothers in the baby care depending on the tolerance of the baby are recommended.

\section{Conclusions}

- Mothers suffer significantly high stress levels when their baby needs NICU admission.

- NICU physical environment and alteration in the parental role are the main sources of stress.

- Infant characteristics like gestational age, birth asphyxia and sepsis are related to high stress levels in the mothers.

- Previous baby needing NICU admission and previous neonatal deaths have strong association with high maternal stress levels. 


\section{References}

1. Miles MS, Funk SG, Carlson J. Parental Stressor Scale: Neonatal Intensive Care Unit. Nursing Research 1993; 42: 148-55. http://dx.doi.org/10.1097/000061991993050 00-00005

PMid: 8506163

2. Dudek-Shriber L. Parent stress in the neonatal intensive care unit and the influence of parent and infant characteristics. American Journal of Occupational Therapy 2004; 58(5):509-20. http://dx.doi.org/10.5014/ajot.58.5.509 PMid: 15481778

3. Morgan Busse K. Parent response to stress in the neonatal intensive care unit. Critical Care Nurse 2012; 33(4):52-60. http://dx.doi.org/10.5014/ajot.58.5.509 PMid: 15481778
4. Carter JD, Mulder RT, Darlow BA. Parental Stress in the NICU. The influence of personality psychological pregnant \& family factors. Journal of Personality and Mental Health 2007; 1: 40-50.

http://dx.doi.org/10.1002/pmh.4

5. Frank LS, Cox S, Allen ANS, Winter I. Measuring neonatal intensive care unit related parental stress. Journal of Advanced Nursing 2005; 49(6): 608-15.

http://dx.doi.org/10.1111/j.13652648.2004.0 3336.x

PMid: 15737221

6. Redshow M. Infants in a neonatal intensive care unit, parental response. Archives of Disease in Childhood Fetal \& Neonatal Edition 2005; 90: 109-13.

http://dx.doi.org/10.1136/adc.2003.031641

PMid: 15724032 PMCid: PMC1721867 